NASA Technical Memorandum 105357

ICOMP-91-27; AIAA-92-0053
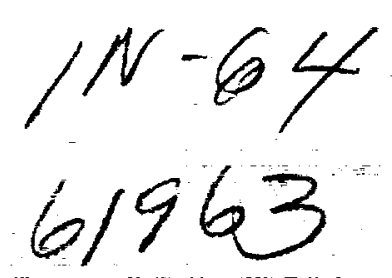

\title{
Central Difference TVD and TVB Schemes for Time Dependent and Steady State Problems
}

\author{
P. Jorgenson \\ Lewis Research Center \\ Cleveland, Ohio
}

and

E. Turkel

Tel-Aviv University

Tel-Aviv, Israel

and Institute for Computational Mechanics in Propulsion

Lewis Research Center

Cleveland, Ohio

Prepared for the

30th Aerospace Sciences Meeting and Exhibit

sponsored by the American Institute of Aeronautics

and Astronautics

Reno, Nevada, January 6-9, 1992

\section{N/SA}
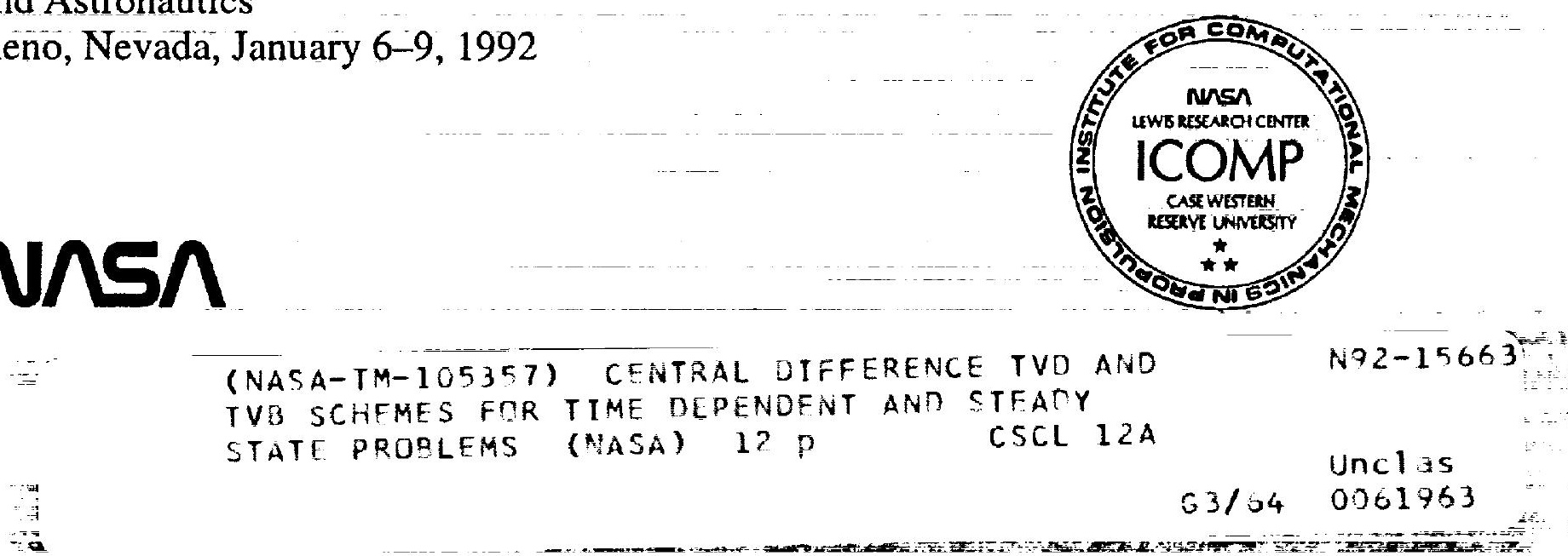


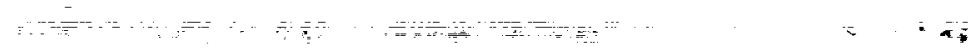




\title{
Central Difference TVD and TVB Schemes for Time Dependent and Steady State Problems
}

\author{
P. Jorgenson" \\ National Aeronautics and Space Administration \\ Lewis Research Center \\ Cleveland, Ohio $\mathbf{4 4 1 3 5}$ \\ E. Turkel ${ }^{\dagger}$ \\ Tel-Aviv University \\ Tel-Aviv 69978, Israel \\ and Institute for Computational Mechanics in Propulsion \\ Lewis Research Center \\ Cleveland, Ohio 44135
}

\section{Abstract}

We use central differences to solve the time dependent Euler equations. The schemes are all advanced using a Runge-Kutta formula in time. Near shocks a second difference is added as an artificial viscosity. This reduces the scheme to a first order upwind scheme at shocks. The switch that is used guarantees that the scheme is locally TVD. For steady state problems it is usually advantageous to relax this condition. Then small oscillations do not activate the switches and the convergence to a steady state is improved. To sharpen the shocks different coefficients are needed for different equations and so a matrix valued dissipation is introduced and compared with the scalar viscosity. The connection between this artificial viscosity and flux limiters is shown. Any flux limiter can be used as the basis of a shock detector for an artificial viscosity. We compare the use of the van Leer. van Albada. minmod. superbee and the "average" flux limiters for this central difference scheme. For time dependent problems we need to use a small enough time step so that the CFL was less than one even though the scheme was linearly stable for larger time steps. Using a TVB Runge-Kutta scheme yields minor improvenents in the accuracy.

\footnotetext{
- Aerospace Engineer. Member AIAA

†Professor. Meniber AIAA

${ }^{0}$ Copyright (C) 1991 by the American Institute of Acronautics and astronautics. Inc. No copyright is asserted in the United States under Title 17. U.S. code. The U.S. Government has a royalty free license to exercise? all rights under the copyright claimed herein for Government purposes. All other rights are reserved by the copyright owntr.
}

\section{Basic Scheme}

The basic elements of the scalar dissipation model considered in this paper were first introduced by Jameson. Schmidt. and Turkel [2] using an explicit Runge-Kutta time integration scheme. The space discretization is based on central differences with an additional artificial viscosity. In this section the basic scheme is briefly reviewed.

Consider the Euler equations in the form

$$
W_{t}+f_{z}=0
$$

where $W$ is the three-component vector of conserved variables. and $f$ is the flux vector. The independent variables are time $t$ and Cartesian coordinate $x$. In a cell-centered. finite-volume method. (1) is integrated over an elemental volume in the discretized computational domain. Equation (1) can also be written as

$$
W_{t}+A W_{x}=0
$$

where $A$ is the flux Jacobian matrix defined by $A=$ $\partial f / \partial W$.

To advance the scheme in time we use a multistage scheme. A typical step of a Runge-Kutta approximation to (1) is

$$
W^{(k)}=W^{(0)}-\alpha_{k} \frac{\Delta t}{\Delta x}\left[D f^{(k-1)}-A V\right],
$$

where $D$ is the spatial differencing operator, and $A V$ represents the artificial dissipation terms. The derivatives of the fluxes are approximated by central differences. In the form presented here the scheme can not have greater than second order accuracy in 
time for nonlinear probloms. For steady state problems the titne accuracy is irrelevant and the form of (2) requires only two levels of storage. If one wishes to obtain higher accuracy in time for nonlinear problems. then one can use any formula from standard numerical ODE theory. In particular the classical Runge-Kutta scheme will give fourth order accuracy using four stages but will require more storage than (2). We will also consider Runge-Kutta forms that preserve the TVD nature of the scheme when the spatial operator is TVD [4].[5]. In all cases. the spatial accuracy is determined only by the accuracy of the operator $\mathrm{D}$ to the derivative.

The dissipation terms are a blending of second and fourth differences. That is.

$$
A V=\left(D^{2}-D^{4}\right) W
$$

where

$$
\begin{gathered}
D^{2} W=\nabla\left[\left(\lambda_{i+\frac{1}{i}} \epsilon_{i+\frac{1}{i}}^{(2)}\right) \Delta\right] W_{i} . \\
D^{4} W=\nabla\left[\left(\lambda_{i+\frac{1}{2}} \epsilon_{i-\frac{1}{2}}^{(i)}\right) \Delta \nabla \Delta\right] W_{i} .
\end{gathered}
$$

and $\Delta . \nabla$ are the standard forward and backward difference operators respectively. The variable scaling factor $\lambda$ is chosen as

$$
\lambda_{i+\frac{1}{2}}=\frac{1}{2}\left[\lambda_{i}+\lambda_{i+1}\right] .
$$

where $\lambda_{i}$ is proportional to the wave speed. The coefficients $\epsilon^{(2)}$ and $\epsilon^{(t)}$ are adapted to the flow and are defined as follows:

$$
\begin{gathered}
\epsilon_{i+\frac{1}{2}}^{(2)}=\kappa^{(2)} \max \left(\nu_{i}, \nu_{i+1}\right) . \\
\epsilon_{i+\frac{1}{2}}^{(1)}=\max \left[0 \cdot\left(\kappa^{(t)}-\epsilon_{i+\frac{1}{i}}^{(2)}\right)\right] .
\end{gathered}
$$

where $\kappa^{(2)}$ and $\kappa^{(t)}$ are constants to be specified.

The parameter $\nu$ is a shock detector. We shall analyze ways of defining $\nu$ in detail in the next section. The purpose of this second difference viscosity is to introduce an entropy-like condition and to suppress oscillations in the neighborhood of shocks. Ideally the value of $\nu$ should be one at shocks and be negligible in smooth regions of the flow. The fourth-difference dissipation term is basically linear and is included to damp high-frequency modes and allow the scheme to approach a steady state. Only this term affects the linear stability of the scheme. Near shocks it is reduced to zero. For time dependent flows. the fourth order dissipation is not very important and $k^{(t)}$ will usually be small or zero.

\section{Shock detectors and flux limiters}

In order to sec the effect of $\nu$ wo first define

$$
\phi_{i}=1-\nu_{i} .
$$

As shown in [6] $\phi$ can be interperted as a flux limiter. though its properties for central difference schemes is slightly different than for upwind schemes. The value of $\phi$ is usually taken as a function of $r$ where

$$
\tau=\frac{u_{i}-u_{i-1}}{u_{i+1}-u_{i}}=\frac{\Delta_{-}}{\Delta_{+}} .
$$

According to the TVD theory for a scalar equation in one dimension the artificial viscosity can sometimes be negative, see (20). However, for multidimensional vector equations with central differences we prefer to be conservative and choose the artificial viscosity, $\epsilon^{(2)}$, to be positive and so we set

$$
\nu_{i}=\left|1-\phi_{i}\right| \text {. }
$$

For the fluid dynamic equations we choose the pressure as a representative of $u$. The artificial viscosity used in the original algorithm was

$$
\nu_{i}=\left|\frac{p_{i+1}-2 p_{i}+p_{i-1}}{p_{i+1}+2 p_{i}+p_{i-1}}\right|,
$$

and $\nu_{i+1 / 2}=\max \left(\nu_{i}, \nu_{i+1}\right)$. We note that with this definition of $\nu$ that $\phi$ is not a function of $r$. We shall demonstrate in the result section that this switch gives rise to oscillations in the flow field.

In order to connect this artificial viscosity with flux limiters we first consider the van Leer flux limiter given by

$$
\phi_{i}(r)=\frac{r+|r|+\varepsilon}{1+|r|+\varepsilon} .
$$

Where $\varepsilon$ is added to prevent the switch from being activated by noise. This $\varepsilon$ is mainly needed for steady state calculations. Then after multiplying (11) by $\left|\Delta_{+}\right|$we get

$$
1-\phi_{i}(r)=\frac{\left(\Delta_{+}-\Delta_{-}\right) \operatorname{sgn}\left(\Delta_{+}\right)}{\left|\Delta_{-}\right|+\left|\Delta_{+}\right|+\varepsilon\left|\Delta_{+}\right|}
$$

Reverting back to the notation of pressure and modifying the $\varepsilon$ term we get

$$
\nu_{i}=\left|1-\phi_{i}(\tau)\right|=\frac{\left|p_{i+1}-2 p_{i}+p_{i-1}\right|}{\left|p_{i+1}-p_{i}\right|+\left|p_{i}-p_{i-1}\right|+\varepsilon},
$$

For dimensional consistency we wish to choose $\varepsilon$ to depend on the pressure. So we choose $\varepsilon=\epsilon\left(p_{i+1}+\right.$ $\left.2 p_{i}+p_{i-1}\right)$. 
Honce.

$\nu_{i}=\frac{\left|p_{i+1}-2 p_{i}+p_{i-1}\right|}{\left|p_{i+1}-p_{i}\right|+\left|p_{i}-p_{i-1}\right|+\epsilon\left(p_{i+1}+2 p p_{i}+p_{i-1}\right)}$

There is no special necd to base the artificial viscosity on the van Leer flux limiter. It is just coincidental that the resultant viscosity $\nu$ closely resembles the original artificial viscosity. (10). Another alternative is the van Albada flux limiter.

$$
\phi_{i}(r)=\frac{r+|r|}{1+r^{2}}
$$

We note that this limiter approaches zero for large values of $r$. while most limiters approach 2 as $r$ increases. Using a similar derivation we find that the artificial viscosity associated with the van Albada limiter is given by

$\nu_{i}=\frac{\left(p_{i+1}-2 p_{i}+p_{i-1}\right)^{2}}{\left(p_{i+1}-p_{i}\right)^{2}+\left(p_{i}-p_{i-1}\right)^{2}+\epsilon\left(p_{i+1}+2 p_{i}+p_{i-1}\right)^{2}}$

There is a second version of the van Albada flux limiter used in the literature.

$$
\phi_{i}(r)=\frac{r+r^{2}}{1+r^{2}}, \quad 0 \leq r \leq 1
$$

Other limiters used are minmod

$$
\phi_{i}(r)=\max (\min (r, 1) .0)
$$

and superbee

$$
\phi_{i}(r)=\max (\min (2 r .1), \min (r .2) .0)
$$

see $[7]$.

We shall also consider the "average" flux limiter

$$
\phi_{i}(r)=\operatorname{minmod}((1+r) / 2.2 \operatorname{minmod}(1 . r)) .
$$

For each of these limiters there is a corresponding "artificial" viscosity.

For an upwind flux limiter we have $\phi\left(\frac{1}{r}\right)=\frac{1}{r} \phi(r)$. Huynh [1] has shown that the resultant scheme is second order if $\phi^{\prime}(1)=1 / 2$. However. for a central difference scheme we have

$$
\phi_{\text {central }}=1-\nu_{i}=1-\left|1-\phi_{i}\right|
$$

and so

$$
\phi_{\text {central }}=\left\{\begin{array}{lll}
\phi_{i} & \text { if } \phi<1 & . r<1 \\
2-\phi_{i} & \text { if } \phi>1 & . r>1
\end{array}\right.
$$

With the van Leer and first Van Albadal flux limiters one finds that $\phi\left(\frac{1}{r}\right)=\phi(r)$. i.e. it doesn't make any difference in (8) if $\mathrm{r}$ is a forward difference over a backward difference or a backward difference over a forward difference. For smoothness we now want $\phi^{\prime}(1)=0$. Of the above limiters only the first version of Van Albada and superbee have this property. It follows from the analysis of [1] that an upwind sclieme can be considered as a symmetric interpolation followed by a upwind convection operator. A central difference scheme can be represented as a downwind interpolation followed by a compensating upwind convection and so the total operation is symmetric.

\section{The TVD Property}

Consider the one-dimensional scalar conservation law

$$
\frac{\partial}{\partial t}[u(x, t)]+\frac{\partial}{\partial x}[f(u(x, t))]=0
$$

where

$$
-\infty<x<\infty, \quad t \geq 0
$$

Let $v(t)=\left\{v_{j}(t)\right\}$ be the approximate solution of (17) and consider the semidiscrete equation 18

I

$$
\begin{array}{r}
\frac{d}{d t} v_{i}(t)+\frac{1}{2 \Delta x}\left[f_{i+1}-f_{i-1}\right]= \\
\frac{1}{2 \Delta x}\left[Q_{i+\frac{1}{2}} \Delta v_{i+\frac{1}{2}}-Q_{i-\frac{1}{2}} \Delta v_{i-\frac{1}{2}}\right] \\
-\frac{\kappa^{(4)}}{\Delta x}\left[R_{i+\frac{1}{2}} \Delta^{3} v_{i+\frac{1}{2}}-R_{i-\frac{1}{2}} \Delta^{3} v_{i-\frac{1}{2}}\right]
\end{array}
$$

with

$$
\Delta v_{i+\frac{1}{2}}=(\Delta v)_{i+\frac{1}{2}}=v_{i+1}(t)-v_{i}(t) .
$$

$\Delta^{3}$ is a third-difference operator defined as

$$
\Delta^{3} v_{i+1}=v_{i+2}(t)-3 v_{i+1}(t)+3 v_{i}(t)-v_{i-1}(t) .
$$

The terms on the right hand side of (18) represent second- and fourth-difference numerical dissipation terms, with $\kappa^{(4)}$ a constant. Define

$$
s_{i+\frac{1}{2}}=\operatorname{sgn}\left(\Delta v_{i+\frac{1}{2}}\right) \text {. }
$$

where sgn represents the signum function. We first shift the indices by one in (18) and subtract (18) from the resulting equation. We then multiply the result by $s_{i+\frac{1}{2}}$ and sum over all $i$. Noting that $s_{i+\frac{1}{2}}= \pm 1$, so $s_{i \div 1}^{2}=1$. and

$$
s_{i+\frac{1}{2}} \Delta v_{i+\frac{1}{2}}=\left|\Delta v_{i+\frac{1}{2}}\right| .
$$

Let $\mathrm{TV}$ denotes the total variation as given by

$$
\mathrm{TV}=\sum_{i}\left|\Delta u_{i+\frac{1}{j}}\right| \text {. }
$$


we then obtain

$$
\begin{aligned}
& \frac{d}{d t} \sum_{i}\left|\Delta v_{i+\frac{1}{2}}\right|= \\
& \quad-\frac{1}{2 \Delta x} \sum_{i} s_{i+\frac{1}{2}}\left(s_{i-\frac{1}{2}}-s_{i+\frac{1}{2}}\right) \frac{\Delta f_{i+\frac{1}{2}}}{\Delta y_{i+\frac{1}{3}}}\left|\Delta v_{i+\frac{1}{2}}\right| \\
& +\frac{1}{2 \Delta x} \sum_{i} s_{i+\frac{1}{2}}\left(s_{i+\frac{1}{2}}-2 s_{i+\frac{1}{2}}+s_{i-\frac{1}{2}}\right) Q_{i+\frac{1}{2}}\left|\Delta v_{i+\frac{1}{3}}\right| \\
& \quad-\frac{\kappa^{(+1)}}{\Delta x} \sum_{i}\left(s_{i+\frac{3}{2}}-2 s_{i+\frac{1}{2}}+s_{i-\frac{1}{2}}\right) R_{i+\frac{1}{3}} \Delta^{3} v_{i+\frac{1}{2}}
\end{aligned}
$$

We stress that the last term will not help for TVD. Its purpose is to eliminate high frequencies and accelerate convergence to a steady state. Hence, we want this contribution to be zero. This can be accomplished if we demand either

$$
\left\{\begin{array}{l}
s_{i+\frac{3}{3}}-2 s_{i+\frac{1}{2}}+s_{i-\frac{1}{2}}=0 \\
\text { or } \\
R_{i+\frac{1}{2}}=0
\end{array}\right.
$$

We are then left with

$$
\begin{aligned}
& \frac{d}{d t}(T V)= \\
& -\frac{1}{2 \Delta x} \sum_{i}-s_{i+\frac{1}{2}}\left(s_{i+\frac{3}{3}}-s_{i-\frac{1}{2}}\right) \frac{\Delta f_{i+\frac{1}{2}}}{\Delta v_{i+\frac{1}{2}}}\left|\Delta v_{i+\frac{1}{2}}\right| \\
& \quad-s_{i+\frac{1}{3}}\left(s_{i+\frac{3}{3}}-2 s_{i+\frac{1}{2}}+s_{i-\frac{1}{2}}\right) Q_{i+\frac{1}{2}}\left|\Delta v_{i+\frac{1}{2}}\right|
\end{aligned}
$$

Thus, a sufficient condition that the total variation not increase is that each term in the summation of (19) must be positive. This means that the scheme is TVD if

$$
\begin{array}{r}
-s_{i+\frac{1}{2}}\left(s_{i+\frac{1}{3}}-2 s_{i+\frac{1}{2}}+s_{i-\frac{1}{2}}\right) Q_{i+\frac{1}{3}} \geq \\
s_{i+\frac{1}{2}}\left(s_{i+\frac{3}{2}}-s_{i-\frac{1}{2}}\right) \frac{\Delta f_{i+\frac{1}{2}}}{\Delta v_{i+\frac{1}{2}}} .
\end{array}
$$

This is the inequality obtained in [6].

When driving the solution to a steady state one frequently finds that it is not advantageous for the scleme to be TVD. The reason is. that with TVD schemes the switches are frequently being turned on and off due to local noise. For steady state calculations this causes the convergence to halt at some error level and a limit cycle results in which the residual oscillates about some level instead of decreasing. To prevent this from occurring we wish to prevent the switch from being activated for small oscillations or small discontinuities. The inequality (20) was obtained by demanding that the solution be TVD and so each term on the right hand side of (10) was $11 \mathrm{eg}$ ative independent of the size of $\frac{\Delta f_{1+1}}{\Delta v_{1+1}}$. Instead we shall only demand that the solution be total variation bounded (TVB). Now, each term on the right hand side of (10) can be positive as long as it is bounded by a constant times $\left|\Delta v_{i+\frac{1}{2}}\right|$. Since $s_{i+1 / 2}$ is equal to plus or minus one we want

$$
-Q_{i+1 / 2}+\frac{\Delta f_{i+\frac{1}{2}}}{\Delta v_{i+\frac{1}{2}}}<\alpha .
$$

$\alpha$ a positive constant.

We shall choose

$$
Q_{i+1 / 2}=\nu_{i+1 / 2} \frac{\Delta f_{i+1}}{\Delta v_{i+\frac{1}{2}}}
$$

This is similar to $(5,6)$ with $\kappa^{(2)}=1 / 2, \nu_{i+1 / 2}=$ $\max \left(\nu_{i}, \nu_{i+1}\right)$, and $\lambda=\frac{\Delta f_{i+1}}{\Delta v_{i+\frac{1}{2}}}$. We then rewrite (22) as

$$
\left(1-\nu_{i+1 / 2}\right) \frac{\Delta f_{i+\frac{1}{2}}}{\Delta v_{i+\frac{1}{2}}}<\alpha .
$$

This implies that if $\frac{\Delta f_{i+\frac{1}{2}}}{\Delta v_{i+\frac{1}{2}}}$ is small then we don't need to turn on the artificial viscosity parameter $\nu$. Only when $\frac{\Delta f_{i+1}}{\Delta v_{i+\frac{1}{3}}} \rightarrow \infty$ do we need that $\nu \rightarrow 1$. In a steady state the shock speed, $\frac{\Delta f_{i+\frac{1}{1}}}{\Delta v_{i+\frac{1}{2}}}$ is zero and so (23) is satisfied for any positive $Q$. So for a steady state scalar equation the TVD property is trivial. Hence. for a steady state problem we do not need the complete TVD theory. However, in this case the solution is also trivial. Moreover, the theory for systems is still not adequate for our purposes. Alternatively, we choose $\nu$ to depend on the strength of the shock, $\Delta v$. For weak shocks $\Delta v$ is small and we can choose $\varepsilon$ near one. For strong shocks $\Delta v$ is large and we want $\varepsilon$ to be small so that $\nu$ is a TVD switch. For the fluid dynamic equations we replace the vector $v$ by the scalar pressure, $\bar{p}$.

To find such a $\nu$ we use (13) When $\epsilon=0$ we get the TVD switch (13) while with $\epsilon=1$ we obtain a perturbation of the original switch, (10) for transonic flows. This switch treats the two sides of the shock asymmetrically depending on whether $p_{i}$ is to the left or right of the shock. Thus, we replace it by

$$
\nu_{i}=\frac{\left|p_{i+1}-2 p_{i}+p_{i-1}\right|}{\left|p_{i+1}-p_{i}\right|+\left|p_{i}-p_{i-1}\right|+\epsilon \max \left(p_{i+1}, p_{i}, p_{i-1}\right)}
$$

In practice the switch that we use is

$$
\nu_{i}=\frac{\left|p_{i+1}-2 p_{i}+p_{i-1}\right|}{(1-\epsilon)\left(\left|p_{i+1}-p_{i}\right|+\left|p_{i}-p_{i-1}\right|\right)+\epsilon\left(p_{i+1}+2 p_{i}+p_{i-1}\right)}
$$


We wish to choose $\epsilon$ antomatically based on the shock strength. One possibility for $\epsilon$ is

$$
\epsilon_{i}=\left\{\frac{\min \left(p_{i-2} \cdot p_{i-1} \cdot p_{i} \cdot p_{i+1} \cdot p_{i-2}\right)}{\max \left(p_{i-2} \cdot p_{i-1} \cdot p_{1} \cdot p_{i+1} \cdot p_{i-2}\right)}\right\}^{\sigma} .
$$

where $\sigma$ is a free parameter. A reasonable range is $\sigma=1 / 2$ to $\sigma=1$.

For small oscillations $p_{i}$ doesn ${ }^{\prime t}$ vary much and so $\epsilon$ is slightly less than 1 . For large oscillations $\epsilon$ is equal to the relative jump across the shock. Consider a perfectly resolved discontinuity going from $p_{L}$ to $p_{R}$ with $p_{R}<p_{L}$. Then.

$$
\nu=\frac{p_{L}-p_{R}}{\left(p_{L}-p_{R}\right)+\epsilon p_{L}} . \quad \epsilon=\left\{\frac{p_{R}}{p_{L}}\right\}^{\sigma} .
$$

Let.

$$
q=\frac{p_{R}}{p_{L}}
$$

Combining these we find that.

$$
\nu=\frac{1}{1+\frac{q^{\sigma}}{1-q}} .
$$

Hence. for small q (i.e. for large discontinuities) $\nu \sim$ $1-q^{\sigma}$. Hence for both very weak shocks and very strong shocks the left hand side of (23) is small i.e. $(1-\nu) \Delta v \rightarrow 0$ as $\Delta v \rightarrow 0$ and also as $\Delta v \rightarrow \propto$. This discussion has concentrated on the theoretical basis of the algorithm. In practice the formula (24) is used for transonic, supersonic and hypersonic flow regimes.

In this section we have written the flux limiters and artificial viscosity in terms of the pressure variable which is appropriate for inviscid fluid dynamics. In the next section we shall consider matrix viscosities. With a matrix viscosity one can base the limiter in each characteristic field on a different quantity.

\section{Matrix Viscosity}

In the above discussion we have discussed a scalar equation. In the original algorithm. this procedure was applied to each equation with the same $\epsilon$. The coefficient $\lambda$ in (5) was chosen as equal to the spectral radius $|u|+c$ while $\nu$ was the same switch that depended on the pressure. for all the equations. For time dependent flows this presents several difficulties. first as seen in the result section there is excessive smearing since the same coefficient is used for all waves and is proportional to the fastest wave speed. Second. pressure is continuous across a contact discontinuity and so a pressure based switch will not sense a contact. We therefore replace the scalar dissipation with a matrix dissipation. i.e $\lambda$ in (3.4) is now a matrix valued function. We first define a function of a matrix $A$. We assume that $A$ can be diagonalized so that $T A T^{-1}$ is diagonal. We then define our function as

$$
\begin{array}{r}
f(A)=\lambda_{3} I+\left(\frac{\lambda_{1}+\lambda_{2}}{2}-\lambda_{3}\right)\left[\frac{\gamma-1}{c^{2}} E_{1}+E_{2}\right] \\
+\frac{\lambda_{1}-\lambda_{2}}{2 c}\left[E_{3}+(\gamma-1) E_{4}\right]
\end{array}
$$

where

$$
\begin{gathered}
E_{1}=\left(\begin{array}{ccc}
\frac{u^{2}}{2} & -u & 1 \\
\frac{u^{3}}{2} & -u^{2} & u \\
\frac{H u^{3}}{2} & -u H & H
\end{array}\right), \\
E_{2}=\left(\begin{array}{ccc}
0 & 0 & 0 \\
-u & 1 & 0 \\
-u^{2} & -u & 0
\end{array}\right), \\
E_{3}=\left(\begin{array}{ccc}
-u & 1 & 0 \\
-u^{2} & u & 0 \\
-u H & H & 0
\end{array}\right), \\
E_{4}=\left(\begin{array}{ccc}
0 & 0 & 0 \\
u^{2} & -u & 1 \\
\frac{u^{3}}{2} & -u^{2} & u
\end{array}\right) .
\end{gathered}
$$

Whenever the matrix $A$ can be diagonalized, i.e. $D=T^{-1} A T$ is diagonal then a function of the matrix is defined by $f(A)=T f(D) T^{-1}$, and $f(D)$ is the function $f$ applied to each element of the diagonal of $D$. Let the coefficients $\lambda_{1}, \lambda_{2}$ and $\lambda_{3}$ be functions of the eigenvalues of $A$. If $\lambda_{1}=u+c, \lambda_{2}=u-c, \lambda_{3}=u$ then we recover the matrix $A$. When the $\lambda$ 's are the absolute value of the eigenvalues we obtain the absolute value of the matrix $A$. In general, $\lambda_{1}, \lambda_{2}$ and $\lambda_{3}$ should not be exactly equal to the eigenvalues of $A$ since at sonic points or stagnation points an eigenvalue is zero and hence no artificial viscosity would be added. Hence, the $\lambda$ 's have a lower limit of $0.2|u+c|$. This procedure also allows one to select different switches for each eigenvalue. In particular we shall base the switch for the nonlinear fields, with speeds $\lambda_{1}$ and $\lambda_{2}$ on the pressure. However, the pressure is continuous across a contact discontinuity. Hence. the switch for the linear field. $\lambda_{3}$ is based on the temperature. $T=\frac{p}{\rho}$. Putting these options together we choose the $\lambda$ 's equal to $\epsilon^{(2)}$ and $\epsilon^{(4)}$ times the limited absolute value of the eigenvalues, see $(3,4)$. 


\section{$5 \quad$ Results}

The results were all obtained using a multistage Runge-Kutta sclicme (2) to advance the solution in time. For most of the computational results the original Runge-Kutta coefficients [2] were used, $\alpha_{1}=1 / 4$. $\alpha_{2}=1 / 3, \alpha_{3}=1 / 2 . \alpha_{4}=1$. Shu [4] introduced another set of coefficients to guarantee that the scheme is TVD in time but is only first order accurate. The three stage scheme has coeffients, $\alpha_{1}=1 / 9 . \alpha_{2}=$ $1 / 3 . \alpha_{3}=1$ while the four stage scheme has coefficients. $\alpha_{1}=1 / 16, \alpha_{2}=1 / 6 . \alpha_{3}=3 / 8, \alpha_{4}=1$. The more stages that are used the larger the time step allowed by stability requirements. However. we found that using larger time steps introduced oscillations into the solution. In practice we chose CFL $=$ .75. and so there was no advantage to using the four stage scheme. Shu [5] also introduced higher order schemes for time dependent equations that are still TVB. These schemes can no longer be written in the simple form of (2). Instead each stage requires the use of the dependent variables and fluxes at previous stages and and so more information needs to be stored.

We solve the one dimensional Euler equations in the domain $0 \leq x \leq 10$. The initial conditions are $u=\overline{0} \mathrm{~m} / \mathrm{s} \cdot \bar{T}=300 \mathrm{~K}$ everywhere. The initial pressure is discontinous with a ratio of $p=20$ for $0 \leq x \leq 5$ to 1 for $5 \leq x \leq 10$. The density and total energy are then calculated from the ideal gas law with $\gamma=1.4$.

We first consider the standard central difference algorithm with a scalar viscosity and the original switch (10) and the original Runge-Kutta coefficients with $\mathrm{CFL}=0.75$. The first figure is a plot of density as a function of $x$ at a nondimensional time of 0.2 . Large oscillations appear both between the rarefaction wave and the contact discontinuity and between the contact and the shock. In figure 2 the density is plotted with the standard switch replaced by the van Leer based switch (9.11). The change in the switch has eliminated all oscillations since the scheme is TVD for the scalar case with this switch (6). There are still some small oscillations in the rarefaction and the contact is very smeared. In figure 3 we show the same case using the matrix viscosity. The switch for the nonlinear waves is based on the pressure as before. Since the pressure is continuous across a contact discontinuity the switch for the entropy wave is based on the temperature. though one could also use entropy. The smearing near the contact is considerably reduced but there remains a small glitch near the sonic point. Using the van Albada(1) based switch improves the tratment of the sonic point. The use of superbee for the nonlinear wave introduced new osrillations as seen in figure 4. We conclude that for the central difference schemes superbee should never be used for the nonlinear waves. The results with minmod was similar to the van Leer viscosity but with a slightly less sharp shock. In all cases the head of the rarefaction wave was smeared out. In figure 5 we present the density when superbee is used for the linear wave while van Albada(1) is used for the nonlinear waves. We also used these schemes with the $\epsilon$ as given in (11). For the van Leer limiter we could choose $\epsilon=.1$ without significantly derading the results while for van Albada(1) we had to choose $\epsilon$ about 0.005 . For the steady problems we can use the van Leer limiter with $\epsilon=.1$ and still get monotone profiles.

The cases presented until now were with the original four stage Runge-Kutta coefficients and CFL = 0.75 . Raising the CFL number introduced oscillations. We next tried the first order scheme suggested by Shu [5] but still got oscillations when the CFL was larger than 1 . We then used the third order Runge-Kutta scheme suggested by Shu [4]. Using the same switches for both the linear and nonlinear switches and these third order Runge-Kutta coeffcients resulted in a sharper profile but some oscillations. Hence, in figure 6 we present the results for Shu's third order scheme in time, using superbee for the linear field and the van Leer viscosity for the nonlinear field. Figure 7 presents the same case as figure 6 but with the CFL raised to 0.95 . This introduced a small oscillation near the sonic point but otherwise was very satisfactory. It is interesting to note that with the scheme of Lerat and Sides [3] the solution becomes less oscillatory as the time step is increased. In our last case we consider the effect of using different variables for the switches. Until now the switch for the nonlinear fields has been based on the pressure while the switch for the linear field has been based on the temperature. We now plot the results when each characteristic field has an artificial viscosity switch based on that characteristic variable. In figure 8 the density is plotted for this case using Shu's third order Runge-Kutta coefficients. $C F L=0.75$, the superbee limiter for the contact discontinuity based on the linearized entropy variable $\Delta p-c^{2} \Delta \rho$, and the van Leer limiter for the acoustic variables $\Delta p+\rho c \Delta u$ and $\Delta p-\rho c \Delta u$. We see that there is an additional improvement in the resolution of the contact discontinuity. The solutions presented are all for the time $t=0.2$. Watching the time evolution one sees that there are many oscillations that occur in the initial breakup of the discontinuity into a shock and a contact. These oscillations disappear as the solution progresses. 
We finally consider a stealy state two dimensional calculation. We solve for the turbulent flow about a blunt cone using a Baldwin-Lomax turbulence model at $M_{x}=25 ., \alpha=0$. The grid is $400 \times 80$. The geometry is shown in figure $9 \mathrm{~d}$. In figure $9 \mathrm{~b}$ we plot the pressure and Mach number along the coordinate line directly in front of the cone. We choose $\epsilon=$ 0.9 in (24). With this high value of $\epsilon$ there are only three points in the shock and no overshoots even at this hypersonic speed. If $\epsilon$ is chosen equal to one (i.e. original switch (10)) the code does not converge. With smaller values of $\epsilon$ the convergence is slowed. In figure $9 \mathrm{c}$ we show the convergence rate for this case on the three grids used in the FMG version of the multigrid.

\section{Conclusion}

- Using a central difference scheme with an artificial viscosity we can duplicate most of the properties of upwind TVD schemes. Solving the one dimensional time dependent Euler equations we get high resolution solutions for the shocks and the contact discontinuity. The main ingredients are an improved shock locator and a matrix artificial viscosity. This shock locator can be based on any of the upwind flux limiters. Superbee is the best for the contact while either van Leer or van Albada(1) is best for the nonlinear waves. Further minor improvements can be obtained by using a high order TVD Runge-Kutta scheme and basing the switch on the characteristic variables. The TVB Runge-Kutta schemes is slightly more accurate than the standard Runge-Kutta schemes.

- There are major differences between the time dependent problem and the steady state problem. For the time dependent problem it was necessary for the scheme to be TVD-like in order to avoid oscillations. However. for the steady state problem we use a coefficient for the artificial viscosity that is considerably below that required for the solution to be TVD and still get monotone shocks even for very strong discontinuities with about four points in the shock layer. Furthermore. high order TVD schemes frequently slow down the convergence to the steady state unless the flux limiters are carefully constructed. When using a TVD scheme coupled with a multigrid acceleration it may be neccessary to limit the transfer of the residual to coarser meshes in the vicinity of shocks. Hence. there is a need for nıre work to extend the TVD theory to steady state problems and also weak shocks.

- For time dependent flows we were not able to use a CFL greater than one even though the lincar stability of the Runge-Kutta allowed larger time steps. For the steady state problems one can use larger time steps. Hence, it is efficient to use many stages in the Runge-Kutta method to increase the CFL stability limit even though one is not interested in high time accuracy. Nevertheless, the limitations on the time step for time dependent problems indicates that even for steady state problems one should limit the local CFL near shocks to less than one at least in the transient phase. This is crucial for hypersonic flow.

\section{References}

[1] Huynh, H. T., Accurate Monotone Cubic Interpolation, NASA TM 103789, March 1991.

[2] Jameson, A., Schmidt, W., and Turkel, E., Numerical Solutions of the Euler Equations by Finite Volume Methods Using Runge-Kutta TimeStepping Schemes, AIAA Paper 81-1259, 1981.

[3] Lerat, A., Sides, J., Efficient Solution of the Steady Euler Equations with a Centered Implicit Method, in "Numerical Methods for Fluid Dynamics, III"', edited by K.W. Morton and M.J. Baines, Clarendon Press, Oxford ,1988, pp. 6586.

[4] Shu, C.-W., Osher, S., Efficient Implementation of Essentially Non-oscillatory Shock-Capturing Schemes, Journal of Comput. Physics, Vol. 77. 1988. pp.439-471.

[5] Shu. C.-W., Total Variation Diminishing Time Discretizations. SIAM J. Sci. Stat. Comput., Vol. 9, 1988. pp. 1073-1084.

[6] Swanson, R. C. and Turkel. E.. On Central Difference and Upwind Schemes, ICASE Report 9044. 1990, to appear in the Journal of Comput. Physics .

[7] Sweby. P.K., High Resolution Schemes Using Flux Limiters for Byperbolic Conservation laws, SIAM Journal of Numerical Analysis, Vol. 21, 1984, pp. 995 - 1011.

[8] Turkel. E.. Improving the Accuracy of Central Difference Schemes, Springer-Verlag Lecture Notes in Physics. 11th International Conference on Numerical Methods in Fluid Dynamics, Vol. 323, 1988, pp. 586-591. 


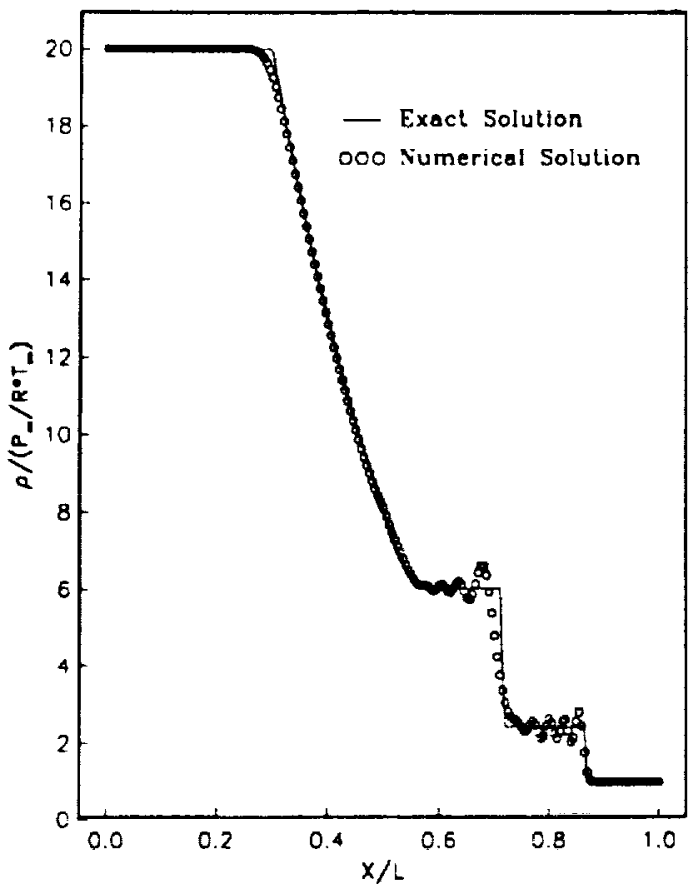

Figure 1: Scalar viscosity with original switch.

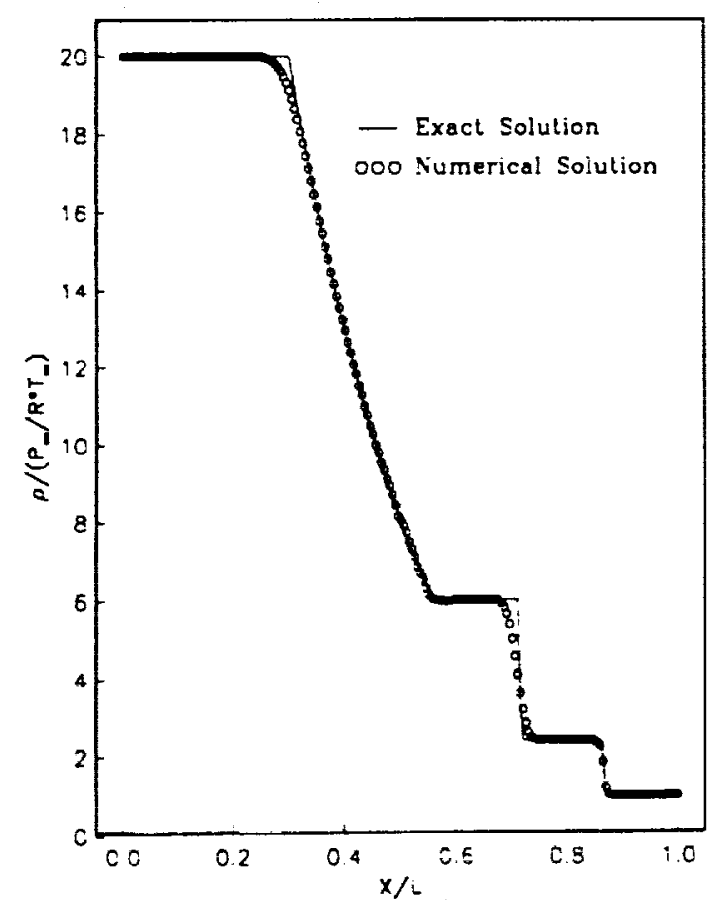

Figure 3: Matrix viscosity with van Leer switch

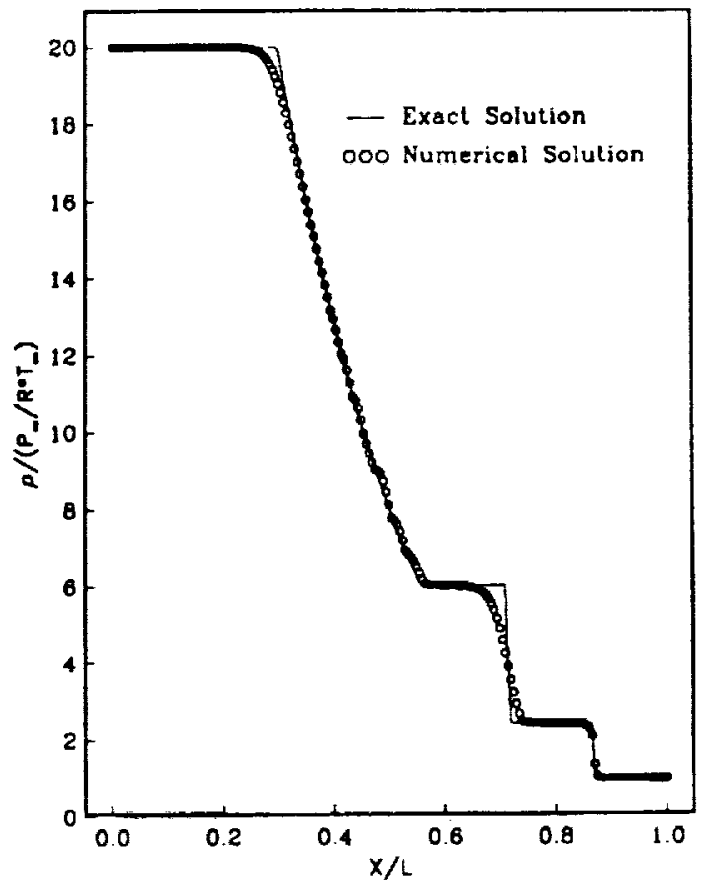

Figure 2: Scalar viscosity with van Leer based switch.

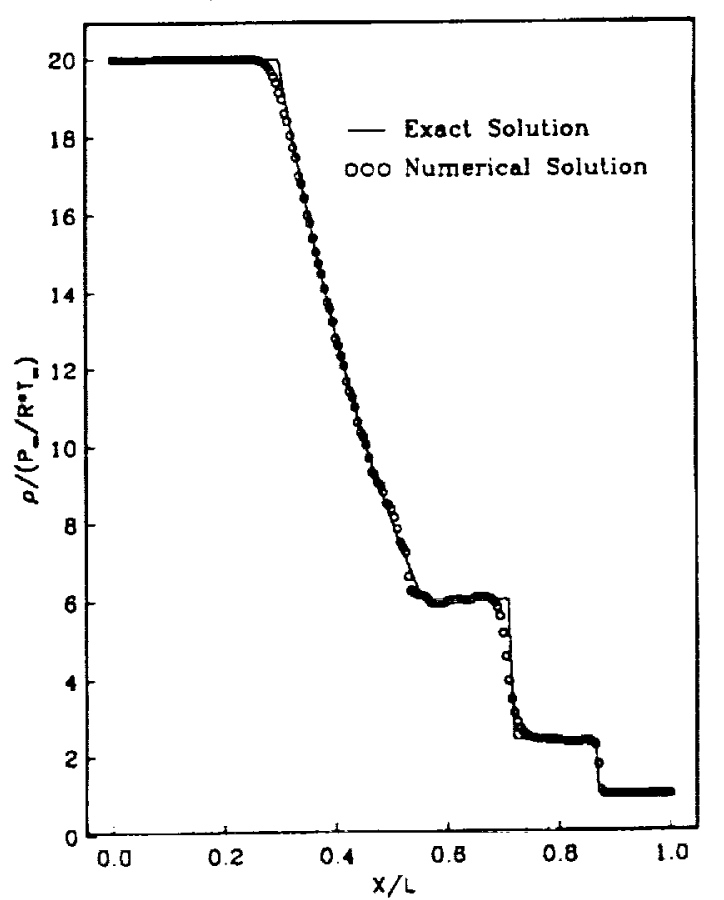

Figure 4: Matrix viscosity with van Albada linear switch and superbee nonlinear swilch. 


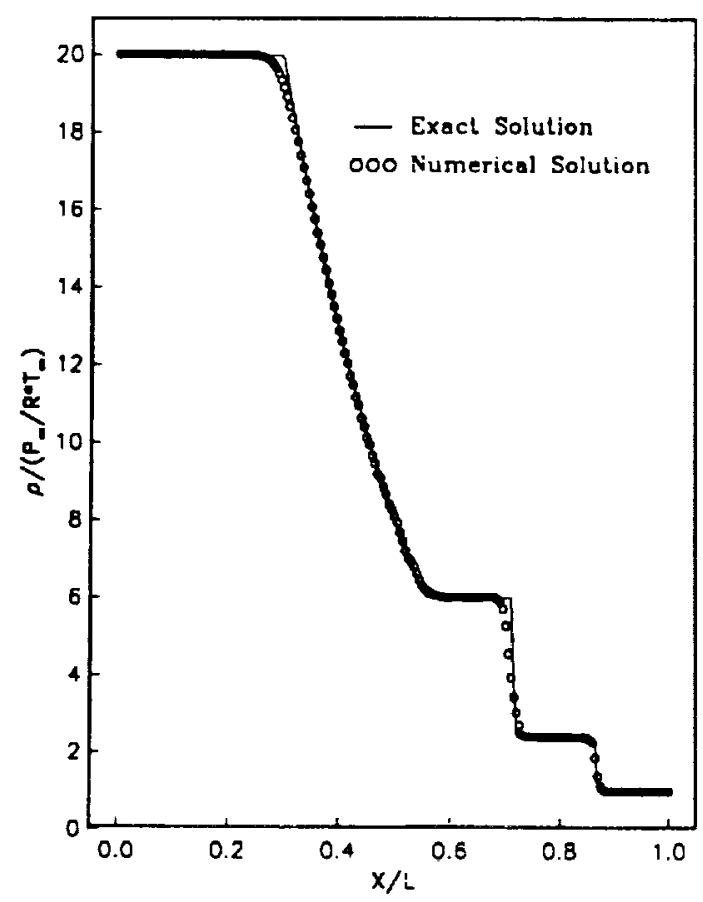

Figure 5: Matrix viscosity with superbee linear switch and van Albada nonlinear switch.

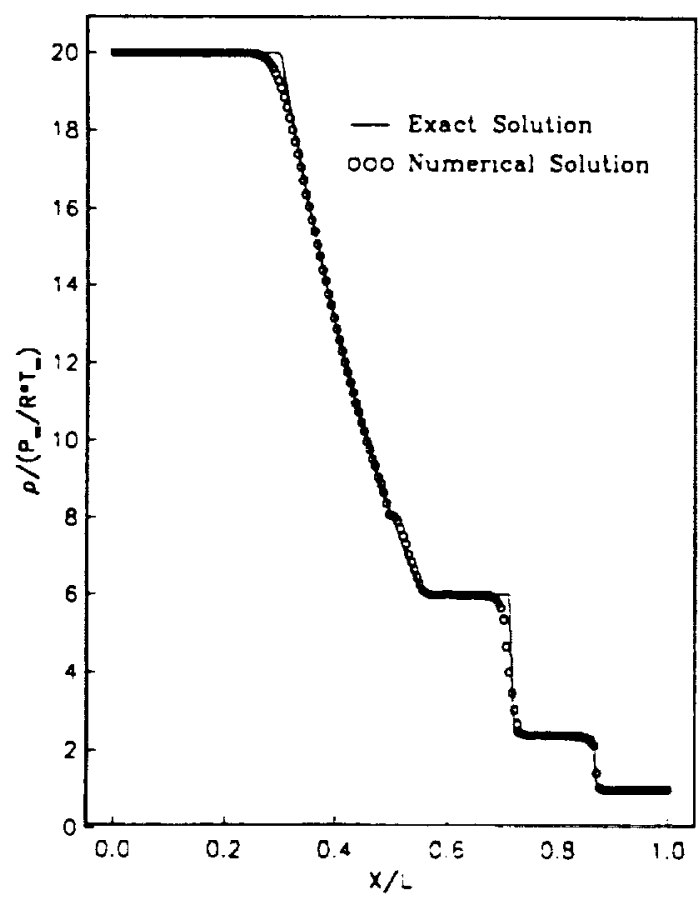

Figure 7: Shu's third order scheme with superbee linear switch and van Leer nonlinear switch, $\mathrm{CFL}=.95$.

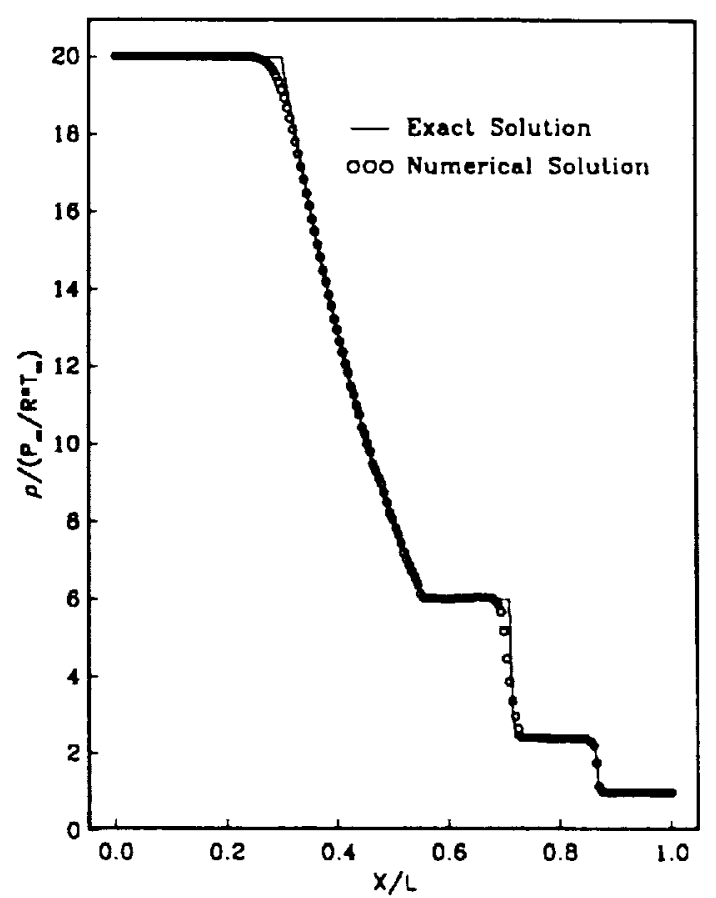

Figure 6: Shu's third order scheme with superbee linear switch and van Leer nonlinear switch, $\mathrm{CFL}=.75$

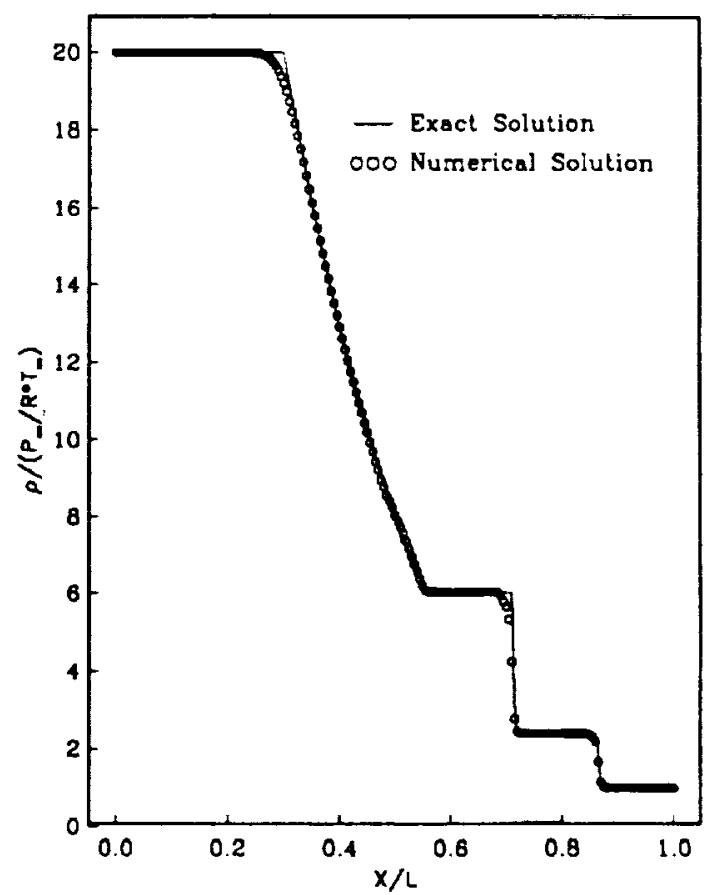

Figure 8: Shu's third order scheme with superbee linear switch and van Leer nonlinear switch based on characteristic variables. 


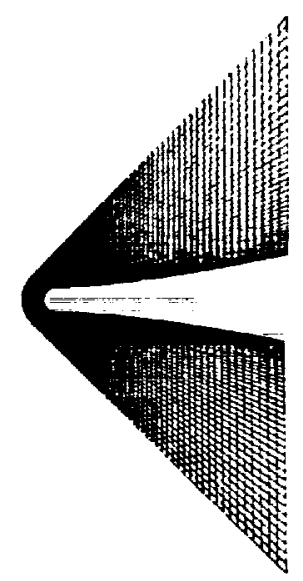

Figre 9a: Geomety tor blunt cone.
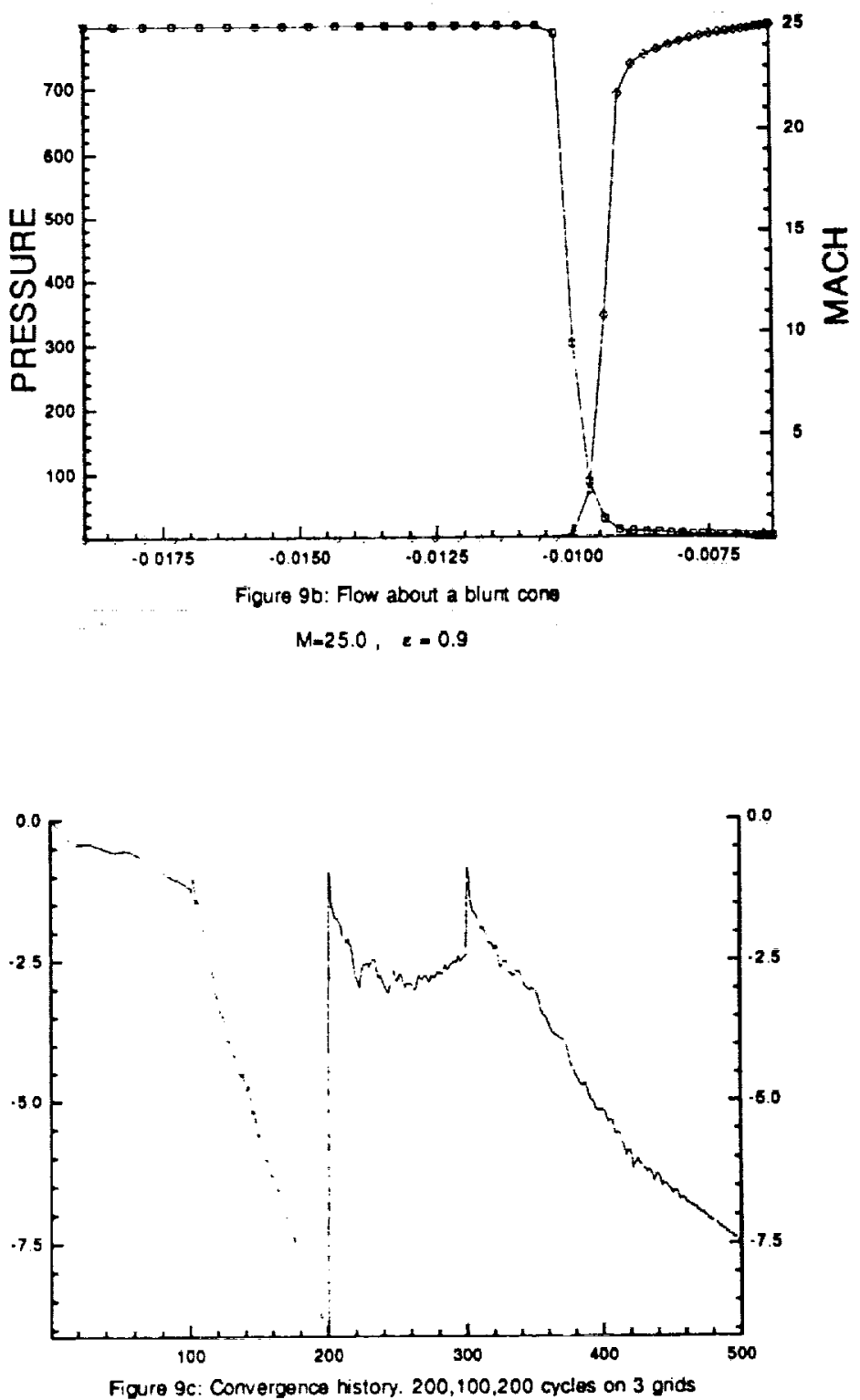



\begin{tabular}{|c|c|c|}
\hline \multicolumn{2}{|c|}{ REPORT DOCUMENTATION PAGE } & $\begin{array}{l}\text { Form Approved } \\
\text { OMB No. } 0704-0188\end{array}$ \\
\hline \multicolumn{3}{|c|}{ 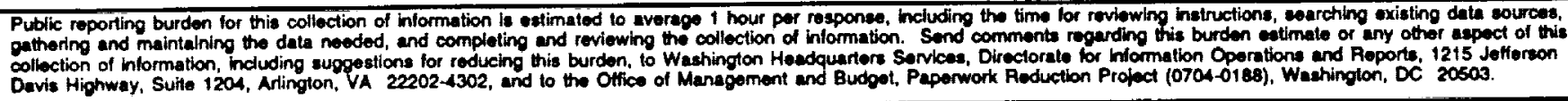 } \\
\hline 1. AGENCY USE ONLY (Leave blank) & $\begin{array}{r}\text { 2. REPORT DATE } \\
1991\end{array}$ & $\begin{array}{l}\text { 3. AEPORT TYPE AND DATES COVERED } \\
\text { Technical Memorandum }\end{array}$ \\
\hline \multicolumn{2}{|c|}{$\begin{array}{l}\text { 4. TITLE AND SUBTITLE } \\
\text { Central Difference TVD and TVB Schemes for Time Dependent } \\
\text { and Steady State Problems }\end{array}$} & \multirow{2}{*}{$\begin{array}{l}\text { 8. FUNDINO NUMBERS } \\
\text { WU-505-62-21 }\end{array}$} \\
\hline \multicolumn{2}{|l|}{$\begin{array}{l}\text { 6. AUTHOA(S) } \\
\text { P. Jorgenson and E. Turkel }\end{array}$} & \\
\hline \multicolumn{2}{|c|}{$\begin{array}{l}\text { 7. PERFORMINO ORGANIZATION NAME(S) AND ADDRESS(ES) } \\
\text { National Aeronautics and Space Administration } \\
\text { Lewis Research Center } \\
\text { Cleveland, Ohio } 44135-3191\end{array}$} & $\begin{array}{l}\text { 8. PEAFOAMING ORGANIZATION } \\
\text { REPORT NUMBER } \\
\text { E-6734 }\end{array}$ \\
\hline \multicolumn{2}{|c|}{$\begin{array}{l}\text { 9. SPONSORING/MONITORING AGENCY NAMES(S) AND ADDRESS(ES) } \\
\text { National Aeronautics and Space Administration } \\
\text { Washington, D.C. } 20546-0001\end{array}$} & $\begin{array}{l}\text { 10. SPONSORING/MONITORING } \\
\text { AGENCY REPOAT NUMBER } \\
\\
\text { NASA TM-105357 } \\
\text { ICOMP }-91-27 \\
\text { AIAA-92-0053 }\end{array}$ \\
\hline
\end{tabular}

11. SUPPLEMENTARY NOTES

Prepared for the 30th Aerospace Sciences Meeting and Exhibit sponsored by the American Institute of Aeronautics and Astronautics, Reno, Nevada, January 6-9, 1992. P. Jorgenson, NASA Lewis Research Center; E. Turkel, Tel-Aviv University, Tel-Aviv, Israel and Institute for Computational Mechanics in Propulsion, NASA Lewis Research Center (work funded under Space Act Agreement C-99066-G). Space Act Monitor, Louis A. Povinelli, (216) 433-5818.

\begin{tabular}{|l|l|}
\hline 12a. DISTRIBUTION/AVALABILITY STATEMENT & 12b. DISTRIBUTION CODE
\end{tabular}

Unclassified - Unlimited

Subject Category 64

13. ABSTAACT (Maximum 200 words)

We use central differences to solve the time dependent Euler equations. The schemes are all advanced using a RungeKutta formula in time. Near shocks a second difference is added as an artificial viscosity. This reduces the scheme to a first order upwind scheme at shocks. The switch that is used guarantees that the scheme is locally TVD. For steady state problems it is usually advantageous to relax this condition. Then small oscillations do not activate the switches and the convergence to a steady state is improved. To sharpen the shocks different coefficients are needed for different equations and so a matrix valued dissipation is introduced and compared with the scalar viscosity. The connection between this artificial viscosity and flux limiters is shown. Any flux limiter can be used as the basis of a shock detector for an artificial viscosity. We compare the use of the van Leer, van Albada, minmod, superbee and the "average" flux limiters for this central difference scheme. For time dependent problems we need to use a small enough time step so that the CFL was less than one even though the scheme was linearly stable for larger time steps. Using a TVB RungeKutta scheme yields minor improvements in the accuracy.

\begin{tabular}{|c|c|c|c|}
\hline \multirow{2}{*}{\multicolumn{3}{|c|}{$\begin{array}{l}\text { 14. SUBJECT TERMS } \\
\text { Euler equations; TVD schemes; Central differences }\end{array}$}} & \multirow{2}{*}{\begin{tabular}{|} 
15. NUMBER OF PAGES \\
12 \\
16. PRICE CODE \\
$\mathrm{A} 03$ \\
\end{tabular}} \\
\hline & & & \\
\hline $\begin{array}{l}\text { 17. SECUAITY CLASSIFICATION } \\
\text { OF REPORT } \\
\text { Unclassified }\end{array}$ & $\begin{array}{l}\text { 18. SECURITY CLASSIFICATION } \\
\text { OF THIS PAGE } \\
\text { Unclassified }\end{array}$ & $\begin{array}{l}\text { 19. SECURTTY CLASSIFICATION } \\
\text { OF ABSTRACT } \\
\text { Unclassified }\end{array}$ & \\
\hline
\end{tabular}

